\title{
Molecular Dynamics Simulations of CRISPR/Cas9
}

\author{
Angana Ray ${ }^{1^{*}}$, Rosa Di Felice ${ }^{l}$ \\ ${ }^{1)}$ University of Southern California, Los Angeles, USA \\ *E-mail anganara@usc.edu
}

Class 2 CRISPR (clustered regularly interspaced short palindromic repeats) systems offer a unique protocol for genome editing in eukaryotic cells and has already been validated in many organisms [1]. In genome editing through CRISPR, the target DNA is first recognized by a complementary RNA bound to an endonuclease. The RNA-guided DNA unwinding leads to formation of a RNA-DNA hybrid duplex and a displaced DNA strand (Figure 1). Thereafter, the nuclease portion of the Cas9 protein closest to the DNA cleavage site attains a conformation essential for concerted DNA cutting.

The nuclease activity of Cas 9 can be also get triggered when there is imperfect complementarity between the RNA guide sequence and an off-target genomic site, which is a major limitation of the CRISPR technique [2] for therapeutic applications. Our goal is to understand

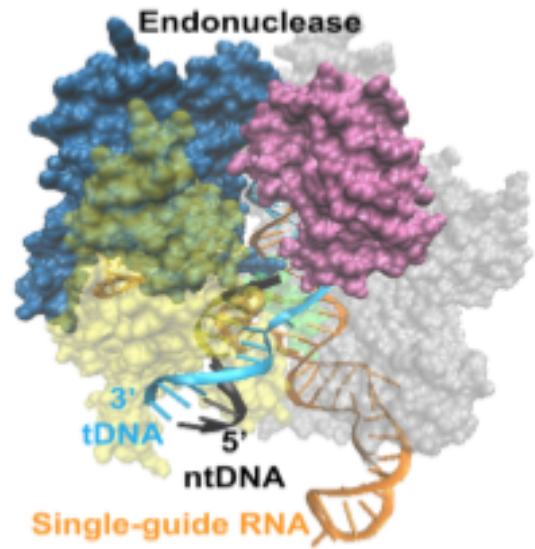

Figure 1. Representation of the crystal structure of a ternary CRISPR/Cas9 complex (PDB ID 4UN3). The cyan and black DNA strands represent the target and non-target DNA strands respectively. The target DNA strand is involved in RNA-DNA hybrid formation with a 20 base long segment of RNA (orange). The protein is represented with surfaces. the binding mechanisms in CRISPR/Cas9 for predicting ways to increase its specificity. We have performed classical MD simulations of CRISPR/Cas9 ternary complexes with the wild type Cas9 [4] and three mutations: K855A, H982A and the combination $\mathrm{K} 855 \mathrm{~A}+\mathrm{H} 982 \mathrm{~A}$, selected from the results of experimental work [3]. Our initial results reveal significant structural impact of the mutations. We find that the unwound part of non-target DNA strand shows highest structural changes for the combination mutation. The distance between the HNH domain of Cas9 and the unwound non-target DNA strand is significantly affected by different mutations, with possible implications for specificity.

\section{References.}

[1] Stella, S; Alcon, P.; Montoya, G. Class 2 CRISPR-Cas RNA-guided endonucleases: Swiss Army knives of genome editing. Nature Struct. Mol. Biol. 2017, 24, 882-892

[2] Zhang, X-H.; Tee, L.Y.; Wang, X-G.; Huang, Q-S.; Yang, S-H.; Off-target Effects in CRISPR/Cas9-mediated Genome engineering. Mol Ther Nucleic Acids. 2015, 4, e264

[3] Slaymaker, I. M.; Gao, L. Y.; Zetsche, B.; Scott, D. A.; Yan, W. X.; Zhang, F. Rationally engineered Cas9 nucleases with improved specificity. Science 2016, 351, 84-88

[4] Tangprasertchai, N.S.; Di Felice, R.; Zhang, X.; Slaymaker, I. M.; Vazquez Reues, C.; Jiang, W.; Rohs, R.; Qin, P. Z. CRISPR-Cas9 Mediated DNA Unwinding Detected Using Site-Directed Spin Labeling. ACS Chem Biol 2017, 12, 1489-1493. 